\title{
Application des modèles de Langmuir et Freundlich aux isothermes d'adsorption des métaux lourds par l'argile purifiée
}

\author{
F. Ayari ${ }^{1,2}$, E. Srasra ${ }^{2}$ et M. Trabelsi-Ayadi ${ }^{1}$ \\ ${ }^{1}$ Laboratoire de Physico-Chimie Minérale, Faculté des Sciences de Bizerte, \\ 7021 Zarzouna-Bizerte, Tunisie \\ ${ }^{2}$ Laboratoire des Matériaux -INRST, BP. 95, 2050 Hammam-Lif, Tunisie
}

\begin{abstract}
Bentonite, which consist essentially of clay minerals belonging to the smectite group, have a wide range of chemical and industrial uses. The structure chemical composition, exchangeable-ion type and small crystal size of smectite are responsible for several properties, including a large chemically active surface area, a high cation-exchange capacity and interlamellar surface having usual hydratation characteristics.

A sample collected from Zaghouan (North East Tunisia, North Africa) is studied through some physicochemical methods. Results from X-ray diffraction, chemical analysis, infrared spectroscopy, thermogravimetric analysis (TGA) and differential thermal analysis (DTA), cation exchange capacities, specific and total surfaces, confirm the general smectite character of the sample.

The adsorption capacity of this clay was tested out using three metallic ions $\left(\mathrm{Pb}^{2+}, \mathrm{Zn}^{2+}, \mathrm{Ni}^{2+}\right)$. The results showed that, in all cases, adsorption can be illustrated by Freundlich or Langmuir isotherms. However, for $10^{-3} \mathrm{M} \mathrm{Pb}^{2+}$ the low value of the correlation coefficient $\left(\mathrm{R}^{2}\right)$ indicated that the experimental data for the adsorption didn't fit to any linear form of the Langmuir equation.

Metal adsorbed onto Zaghouan clay varied in the decreasing order $\mathrm{Pb}^{2+}>\mathrm{Zn}^{2+}>\mathrm{Ni}^{2+}$ and fitted in satisfactorily with the uptake capacity. For $\mathrm{Pb}^{2+}$ the amount of adsorbed ions remained higher than the CEC (cation exchange capacity) of the clay fraction. This result may be due to adsorption of hydroxy lead complex in addition to sorption of bivalent lead form which explains the high amount of $\mathrm{Pb}^{2+}$ removed from aqueous solution.
\end{abstract}

\section{INTRODUCTION}

L'utilisation des argiles comme adsorbants, présente un intérêt dans le traitement des eaux de rejets industriels. Ceci est justifié par l'importance de la surface développée par ce matériau, par la présence de charges négatives sur la surface, par la possibilité d'échange des cations et par une large disponibilité dans la nature.

Une argile purifiée, provenant de la région de Zaghouan (Nord-Est de la Tunisie), est utilisée pour le traitement de purification des eaux souterraines susceptibles d'être polluées par des métaux lourds (Zinc, Plomb, Nickel). L'argile est caractérisée par plusieurs méthodes physico-chimiques, telles que la diffraction des rayons $\mathrm{X}$, la spectroscopie infrarouge, l'analyse thermique et l'analyse chimique.

Les isothermes d'adsorption ont été déterminées à la température ambiante dans les conditions physico-chimiques proches de celles du traitement des eaux industrielles moyennement acides $(\mathrm{pH}=5)$. 


\section{PARTIE EXPÉRIMENTALE}

\section{2 .1 Caractérisation physico-chimique}

A partir de l'échantillon appartenant à la série argileuse d'âge santonien-campanien inférieur (Crétacé supérieur), est récupérée la fraction de granulométrie inférieure à $100 \mu \mathrm{m}$.

Après les étapes de purification [1], l'échange sodique est suivi par diffraction des rayons X. La distance $\mathrm{d}_{001}$ de la smectite, initialement égale à $14 \AA$, diminue jusqu'à atteindre $12 \AA$. Ce traitement de l'échantillon naturellement calcique le rend sodique.

La diffraction des RX révèle que l'échantillon d'argile (ZG2) est composé de smectite, d'illite et d'une faible fraction de kaolinite éliminée après purification et échange sodique (fig-1).

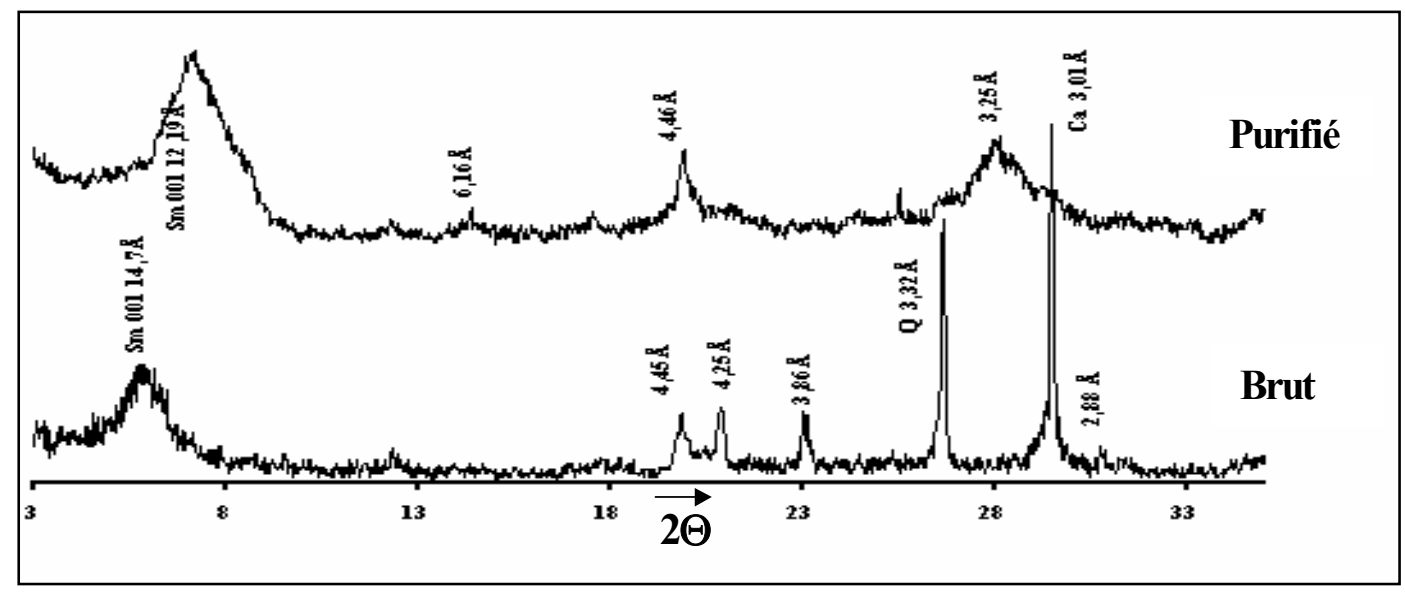

Figure 1. Diffractogrammes des rayons X des échantillons ZG2b (brut) et ZG2p (purifié).

Les compositions centésimales des échantillons ZG2b (brut) et ZG2p (purifié), déterminées par spectrophotométrie d'absorption atomique, sont reportées dans le tableau-1:

Tableau 1. Compositions centésimales (en taux d'oxydes) des échantillons ZG2b et ZG2p.

\begin{tabular}{|l|l|l|l|l|l|l|l|l|l|}
\hline Echantillon & $\mathrm{SiO}_{2}$ & $\mathrm{MgO}$ & $\mathrm{CaO}$ & $\mathrm{Fe}_{2} \mathrm{O}_{3}$ & $\mathrm{Al}_{2} \mathrm{O}_{3}$ & $\mathrm{Na}_{2} \mathrm{O}$ & $\mathrm{K}_{2} \mathrm{O}$ & $\mathrm{PF}^{*}$ & Total \\
\hline ZG2b & 40,96 & 3,43 & 16 & 5,09 & 9,23 & 1,46 & 0,43 & 23,4 & 100 \\
\hline ZG2p & 50,8 & 3,95 & 0,28 & 6 & 17 & 1,39 & 0,84 & 19,74 & 100 \\
\hline
\end{tabular}

PF: Perte au feu.

Le rapport $\mathrm{SiO}_{2} /\left(\mathrm{Al}_{2} \mathrm{O}_{3}+\mathrm{MgO}+\mathrm{Fe}_{2} \mathrm{O}_{3}\right)$ proche de 2 indique que cet argile est un phyllosilicates de type $2 / 1$.

Le taux de fer qui augmente après purification, de $5,09 \%$ à $6 \%$, pourrait indiquer que le fer est contenu dans le réseau argileux.

L'augmentation du taux de $\mathrm{K}_{2} \mathrm{O}$ après purification est probablement dû à l'existence d'illite.

La formule structurale globale de l'échantillon (ZG2p) [2] est :

$\mathrm{Na}_{0,404} \mathrm{Ca}_{0,043} \mathrm{~K}_{0,16}\left(\mathrm{Si}_{7,56} \mathrm{Al}_{0,44}\right)\left(\mathrm{Al}_{2,933} \mathrm{Fe}_{0,678} \mathrm{Mg}_{0,476}\right) \mathrm{O}_{22}$ 
La capacité d'échange cationique est $89 \mathrm{méq} / 100 \mathrm{~g}$ d'argile calcinée, la surface spécifique est $504 \mathrm{~m}^{2} \mathrm{~g}^{-1}$, ces valeurs sont inférieures à celle d'une montmorillonite. Cette différence pourrait être due à la présence d'illite.

Les courbes d'analyse thermogravimétrique (fig-2) permettent de suivre la perte de masse des échantillons. Trois pertes de masse sont observées pour ZG2b et deux seulement pour ZG2p. Les pertes d'eau d'humidité et d'eau interfoliaire se produisent entre 303 et $403 \mathrm{~K}$, la perte d'eau de constitution à $799 \mathrm{~K}$. La décarbonatation de l'argile se produit à 893 et $1073 \mathrm{~K}$. Cette perte n'apparaît pas pour l'échantillon purifié.

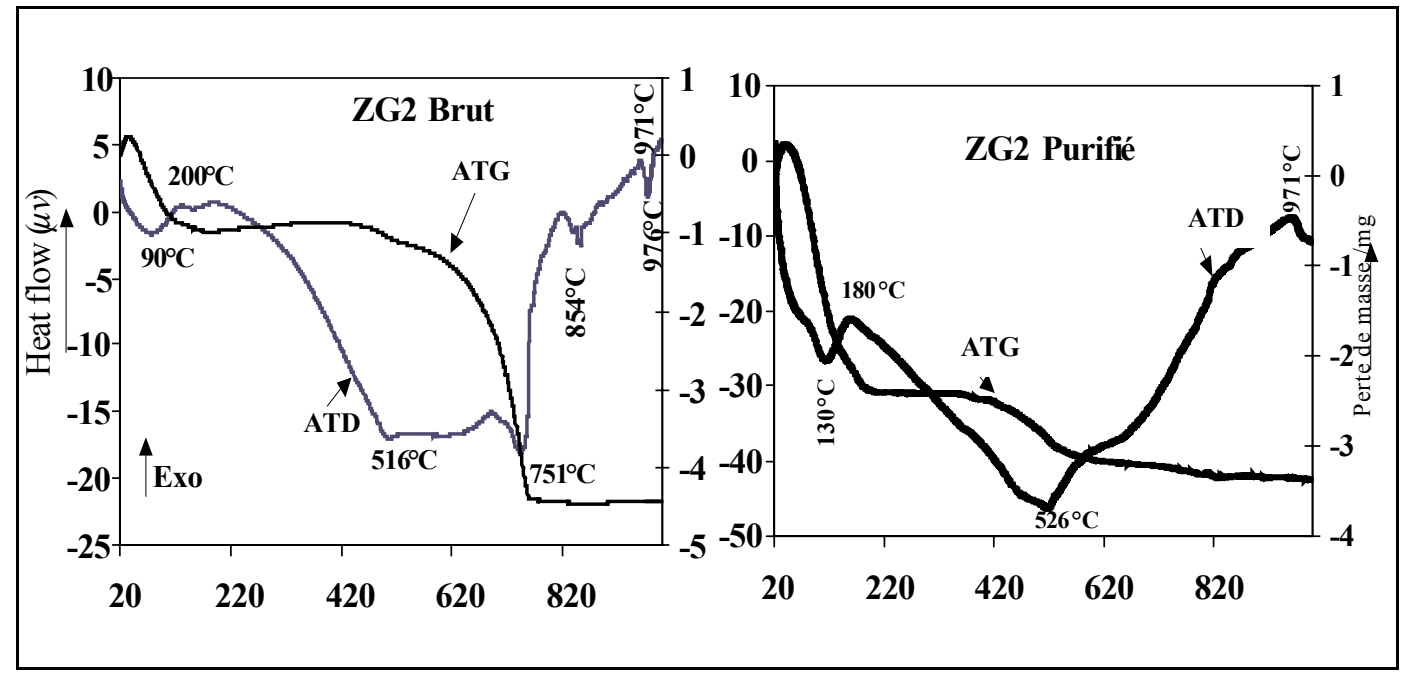

Figure 2. Courbes ATG-ATD des échantillons ZG2b et ZG2p.

Le caractère dioctaédrique [3] de la fraction argileuse et l'identification à une smectite dioctaédrique [4-5] sont confirmés par spectroscopie infrarouge (fig-3) : les bandes de vibration de valence à 3626 $\mathrm{cm}^{-1}$ et de déformation à $910 \mathrm{~cm}^{-1}$ correspondent à la vibration $\mathrm{Al}-\mathrm{Al}-\mathrm{OH}$.

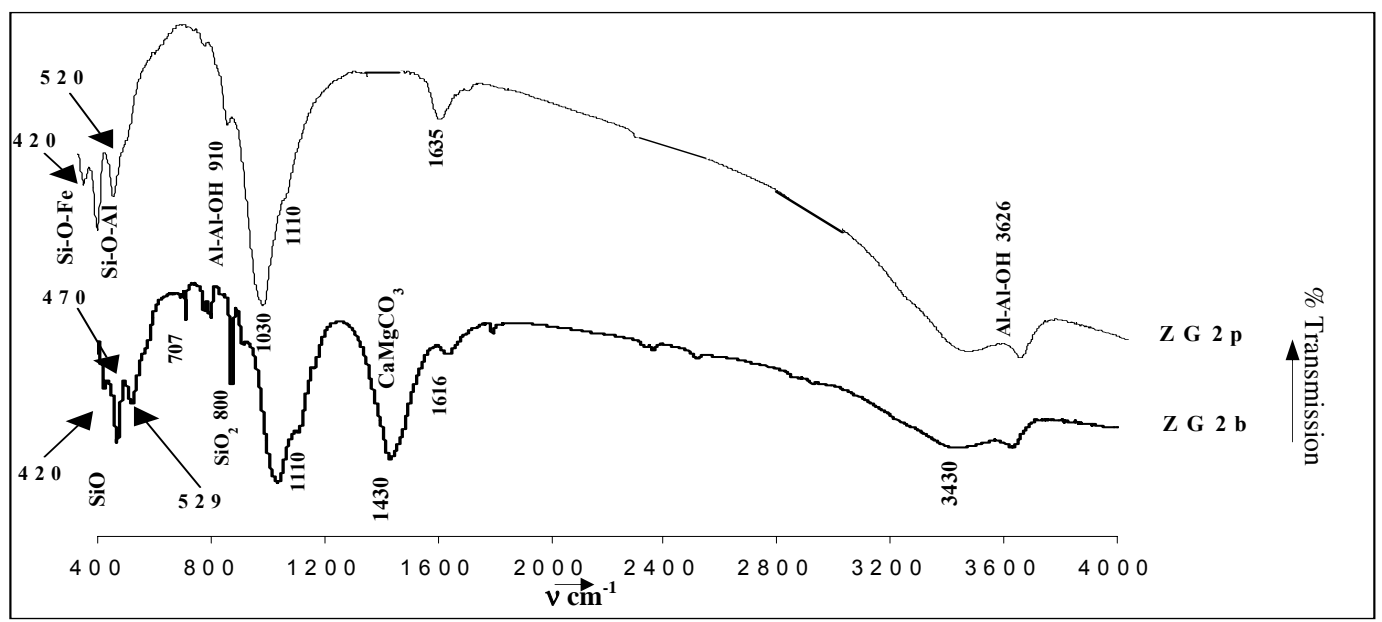

Figure 3. Spectres infrarouges des échantillons ZG2b et ZG2p. 


\subsection{Isothermes d'adsorption}

L'adsorption des cations métalliques $\mathrm{Zn}^{2+}, \mathrm{Pb}^{2+}, \mathrm{Ni}^{2+}$ par l'argile bentonitique (ZG2p sodique) dans les conditions les plus proches du traitement des eaux industrielles moyennement acides $(\mathrm{pH}=5)$ et à température ambiante (294K) [6] est suivie par spectroscopie d'adsorption atomique. L'appareillage utilisé est de marque AAS vario 6 « Analytic jena ».

Trois solutions de sels métalliques de concentrations $\mathrm{x} 10$, x5 et égales à la capacité d'échange cationique de l'échantillon argileux $\left(10^{-2}, 5.10^{-3}, 10^{-3} \mathrm{M}\right)$ ont fait l'objet de cette étude. La variation des concentrations du métal en solution en fonction de la teneur en métal adsorbé dans la fraction argileuse à l'équilibre a été suivie (fig-4) par l'établissement des isothermes d'adsorption.

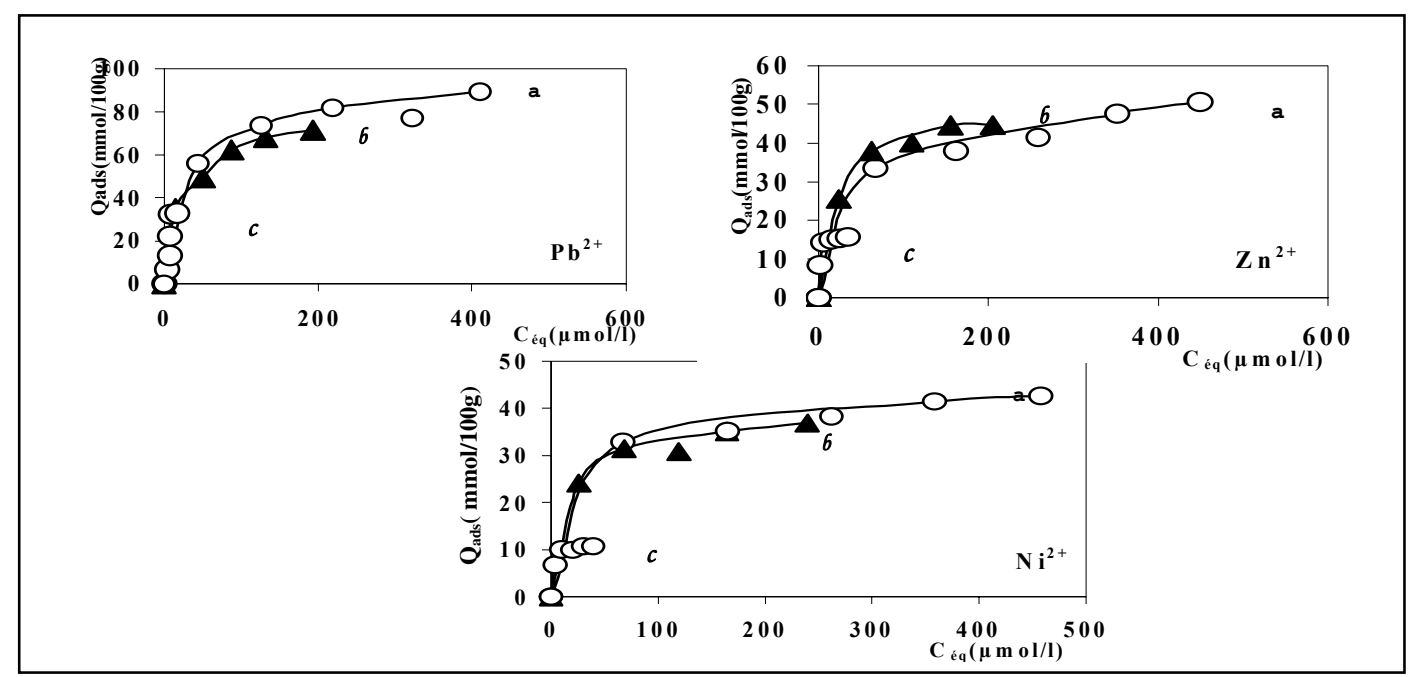

Figure 4. Isothermes d'adsorption de $\mathrm{Pb}^{2+}, \mathrm{Zn}^{2+}, \mathrm{Ni}^{2+}$ par $\mathrm{ZG} 2 \mathrm{p}$ $\mathrm{a}=[\mathrm{Pb}]_{0}=[\mathrm{Zn}]_{0}=[\mathrm{Ni}]_{0}=10^{-2} \mathrm{M} ; \mathrm{b}=[\mathrm{Pb}]_{0}=[\mathrm{Zn}]_{0}=[\mathrm{Ni}]_{0}=5.10^{-3} \mathrm{M} ; \mathrm{c}=[\mathrm{Pb}]_{0}=[\mathrm{Zn}]_{0}=[\mathrm{Ni}]_{0}=10^{-3} \mathrm{M}$.

Le pH final du liquide surnageant est augmenté d'une ou deux unités par rapport à sa valeur initiale. Ceci pourrait indiquer que l'adsorption des protons de la solution métallique, par échange avec les cations de l'argile, est très élevée, ou que cette élévation du $\mathrm{pH}$ est due à la rétention de $\mathrm{H}^{+}$par l'argile. Cela peut entraîner la complexation des métaux ou leur précipitation.

En ce référant au diagramme potentiel - $\mathrm{pH}$ de ces trois cations [7], le nickel et le zinc sont adsorbés sous la forme bivalente $\left(\mathrm{Ni}^{2+}, \mathrm{Zn}^{2+}\right)$ alors que le plomb pourrait apparaître sous la forme monovalente $\mathrm{Pb}(\mathrm{OH})^{+}$et sous sa forme bivalente $\mathrm{Pb}^{2+}$. Ceci a permis d'expliquer les quantités élevées de plomb adsorbées par rapport à la CEC et par rapport à celles des deux autres cations (nickel et zinc). Comparativement, la quantité du plomb adsorbée est supérieure à celle du zinc qui est elle-même supérieure à celle du nickel. Les résultats montrent donc la sélectivité préférentielle suivante : $\mathrm{Pb}>\mathrm{Zn}>\mathrm{Ni}$.

\section{3 Interprétation des résultats expérimentaux selon le modèle de Langmuir et Freundlich}

Les résultats de l'adsorption sont modélisés en s'appuyant sur les hypothèses de Langmuir (fig-5) et Freundlich (fig-6). Les équations qui servent aux modèles sont respectivement: $\mathrm{C} /(\mathrm{x} / \mathrm{m})=1 / \mathrm{a}+\mathrm{b} / \mathrm{a} \mathrm{C}$, et $\mathrm{x} / \mathrm{m}=\mathrm{kC}^{\mathrm{n}}$, dans lesquelles $\mathrm{x} / \mathrm{m}$ représente la quantité de métal adsorbé par unité de masse de l'adsorbant et $\mathrm{C}$ représente la concentration à l'équilibre du cation $\mathrm{M}^{2+}$ exprimée en $\mathrm{gL}^{-1}$, a et $\mathrm{b}$ sont les constantes de Langmuir, $\mathrm{k}$ et $\mathrm{n}$ sont celles de Freundlich. 


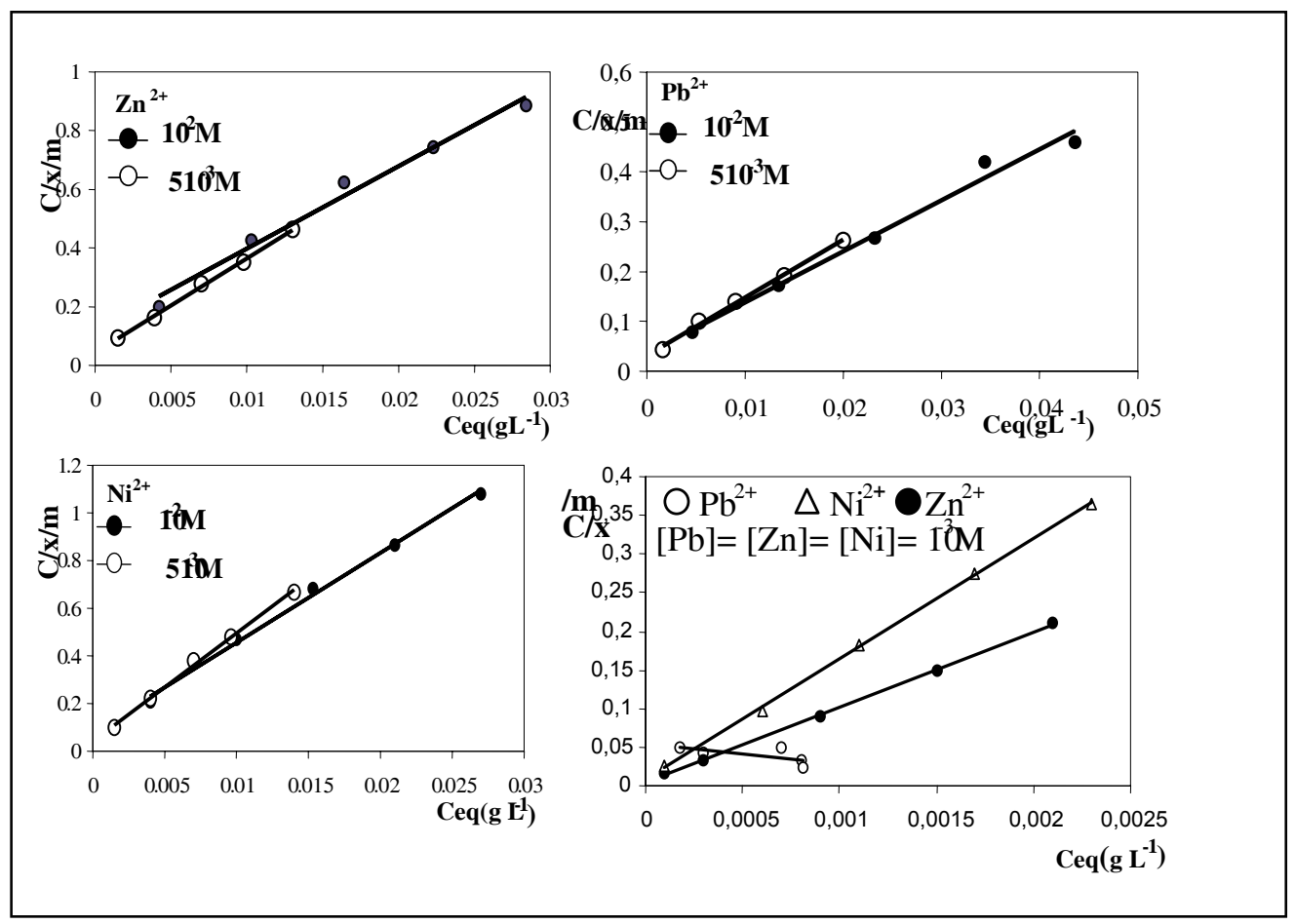

Figure 5. Isothermes d'adsorption de $\mathrm{Pb}^{2+}, \mathrm{Zn}^{2+}$, et $\mathrm{Ni}^{2+}$ par $\mathrm{ZG} 2 \mathrm{p}$ selon le modèle de Langmuir.

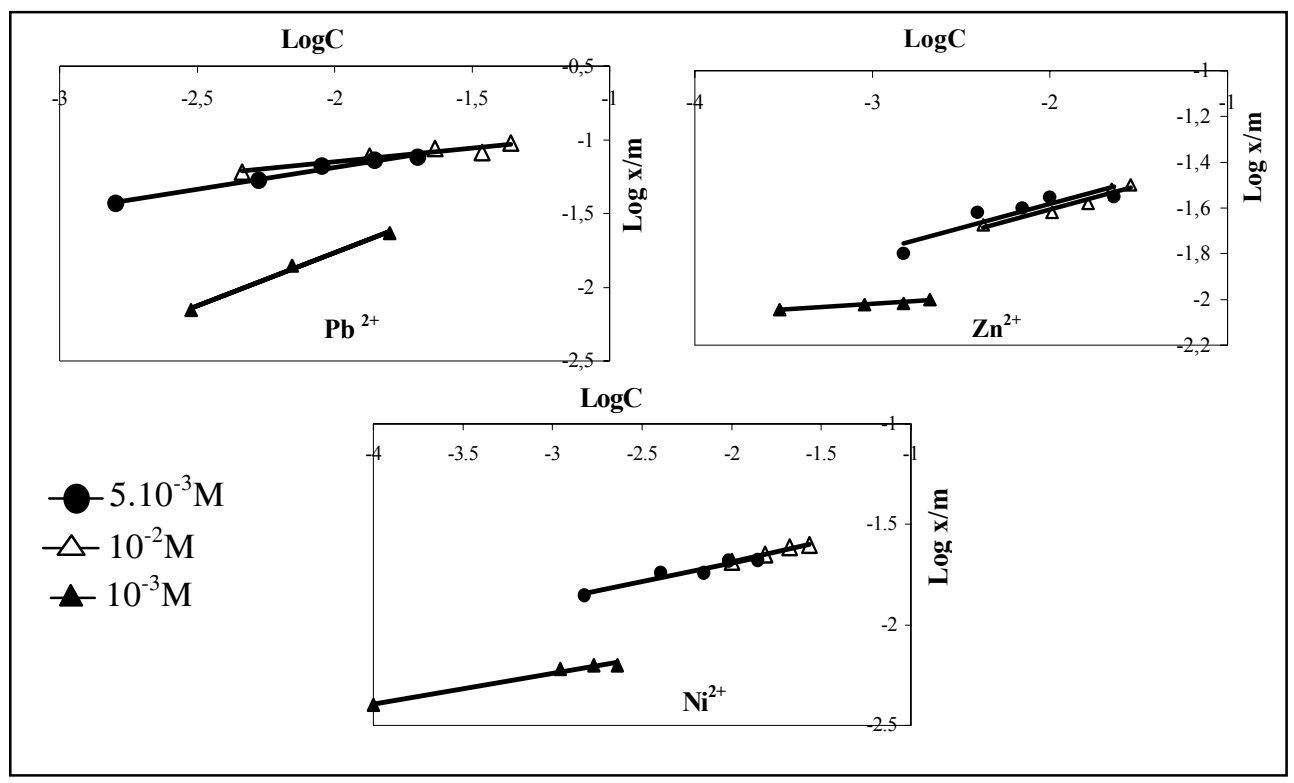

Figure 6. Isothermes d'adsorption de $\mathrm{Pb}^{2+}, \mathrm{Zn}^{2+}$, et $\mathrm{Ni}^{2+}$ par $\mathrm{ZG} 2 \mathrm{p}$ selon le modèle de Freundlich.

La modélisation des résultats expérimentaux montre une bonne concordance avec le modèle de Freundlich. En effet Les coefficients de corrélation trouvés sont tous proches de 0,9 , contrairement à celui de Langmuir avec lequel certains cas ne concordent pas. 


\section{CONCLUSION}

L'adsorption de zinc, nickel et plomb par la bentonite de Zaghouan purifiée est dominée par le phénomène d'échange cationique. Ce phénomène de rétention des cations apparaît avec un ordre préférentiel de sélectivité : $\mathrm{Pb}^{2+}>\mathrm{Zn}^{2+}>\mathrm{Ni}^{2+}$.

L'adsorption des métaux croît avec la concentration du métal en solution, elle atteint ou dépasse légèrement la capacité d'échange cationique lorsque la concentration du métal en solution est $5.10^{-3} \mathrm{M}$. Pour l'étude de la fixation des trois métaux, le modèle de Freundlich donne des résultats plus cohérents que le modèle de Langmuir.

\section{Références}

[1] Van Olphon, An introduction to clay colloid chemistry (Interscience Publishers, NY. London) 1963

[2] Mauguin C, Etude des micas au moyen des RX., Bull .Soc.Fr. Miner 51 (1928) 128-332

[3] Caillere. S; Henin. S et Rautrureau. M, Minéralogie des argiles (Vol I et II, Masson) (1982)

[4] Dixon J.B \& Weed S.B, Minerals in soil environnement (Published by Soil Science Society of America, Madison, Wisconsin, U.S.A) (1977)

[5] Sposito et Prost. R., Structure of water adsorption on smectites, Chem. Rev, P 28 (1982) 553-573

[6] Baccouche.A et Srasra E., Adsorption de $\mathrm{Pb}^{2+}, \mathrm{Cd}^{2+}$ et $\mathrm{Cu}^{2+}$ en solution par la zéoliteNa-P1, Journal de la Société chimique de Tunisie Vol. IV, N8, 837-849, (2000)

[7] Baes. C. F et Mesmer. R. E, The Hydrolyses of cation (Wiley, New York) (1976) 